\title{
Management of bladder stones associated with foreign bodies following incontinence and contraception surgery
}

\author{
Abdulmuttalip Simsek ${ }^{1}$, Faruk Ozgor ${ }^{1}$, Mehmet Fatih Akbulut ${ }^{1}$, Erkan Sönmezay ${ }^{1}$, Bahar Yuksel ${ }^{2}$, \\ Omer Sarılar ${ }^{1}$, Ahmet Yalcın Berberoglu ${ }^{1}$, Zafer Gokhan Gurbuz ${ }^{1}$ \\ ${ }^{1}$ Haseki Research and Education Hospital, Department of Urology, Istanbul, Turkey; \\ ${ }^{2}$ Istanbul Medical Faculty, Gynecology and Obstetric Department, Istanbul, Turkey.
}

\begin{abstract}
Summary Aim of the study: To investigate success of endoscopic lithotripsy for bladder stone following stress urinary incontinance surgery and contraception surgery.

Materials and methods: Charts of patients admitted in two centers between January 2006 and March 2013 were retrospectively reviewed and seven women were enrolled in our study. Patients demographic parameters including age, main complaint(s), previous surgery type, time to diagnosis were analyzed. Also operative time, hospitalisation lenght, perioperative and postoperative complication(s) were evaluated.

Results: Five patients had undergone tension free vaginal tape procedure and one patient had undergone transobturator tape procedure. Median age was 62 (50-71) years. In one patient bladder stone formed around an intrauterine device. Dysuria (85\%), hematuria (57\%) and recurrent urinary tract infection (57\%) were the main complaints. The median diagnosis time was 44.1 months. Abdominal ultrasonography and non contrast enhanced computer tomography were performed for five and two patients respectively and diagnosis was confirmed cystoscopically. Endoscopic lithotripsy using Holmium laser lithotripter or pneumatic lithotripter was used for all cases. The mean operation time was 41.2 minutes (20-70) and success was $100 \%$. There was no intraoperative complication. Only one patient had fever higher than $38^{\circ} \mathrm{C}$ postoperatively and was treated by appropriate antibiotic. The median hospitalisation time was 1.57 day. Conclusion: In conclusion endoscopic lithotripsy is a safe and effective approach to manage bladder stone associated with mid-urethral synthetic slings and intrauterine devices.
\end{abstract}

KEY WORDS: bladder stone; Endoscopic cystolithotripsy; Intrauterine device; Mid urethral synthetic sling.

Submitted 17 September 2013; Accepted 5 October 2013

\section{INTRODUCTION}

Bladder stones (BS) are rare in women and consist $5 \%$ of all cases (1). Generally stone formation in the bladder is

No conflict of interest declared. related with an underlying pathology such as neurogenic bladder, pelvic organ prolapse or foreign bodies (2). Benefits of intrauterin devices (IUD) and mid-urethral synthetic slings (MUSS) were well described in contraception and stress urinary incontinence, respectively (3-4). Migration of IUD and MUSS into the bladder leads to dysuria, urgency, pelvic pain, recurrent urinary tract infection and BS (5). When BS occur, removal of BS and foreign body is mandatory. Several Authors have defined different approaches to solve this medico-legal problem. Despite acceptable success rate of all techniques, open approaches as cystotomy and partial cystectomy are associated with new incision scar, painful post operative period and longer hospitalisation time when compared with endoscopic treatment modalities (6-7).

In this paper we aim to present our experience about BS associated with IUD and MUSS.

\section{Materials AND Methods}

We conducted an observational study through chart review of patients who were treated for BS. From January 2006 to May 2013, eighteen patients were treated for BS in Sultangazi Goverment Hospital and Haseki Training And Research Hospital. Seven women had BS associated with IUD or MUSS and were enrolled in the study. Diagnosis was confirmed by imaging studies (ultrasonography or non-contrast enhanced computer tomography) and endoscopically.

The operative procedure was similar for all patients. Endoscopic cystolithotripsy was perfomed using Holmium-Yag laser or pneumatic lithotripter to expose IUD or MUSS (Figure 1). To disconnect the MUSS from the bladder wall, endoscopic scissors and transurethral resection (TUR) with monopolar diathermy were used. When the foreign body was completely separated from the wall of the bladder, an endoscopic forcep was used to remove the IUD or MUSS.

Patients' age, main complaints, diagnosis method and time from surgery to diagnosis were evaluated. Also operative time, treatment modality and lenght of hospitalization were analyzed. Median diagnosis time was defined as the 
Figure 1.

Bladder stone formed on TVT sling.

Pneumatic lithotripter was used to fragment the stone.

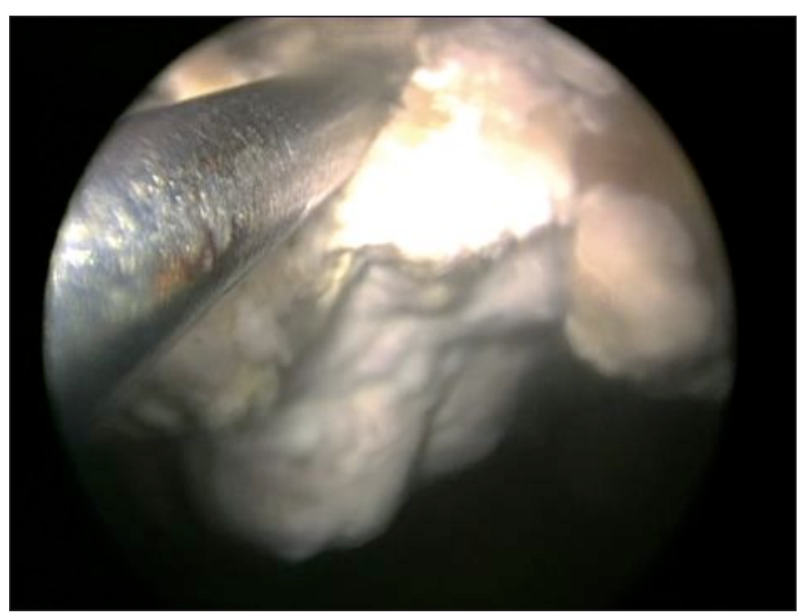

Figure 2.

Image of IUD and fragmented stones after operation. Endoscopic forceps was used to remove IUD.

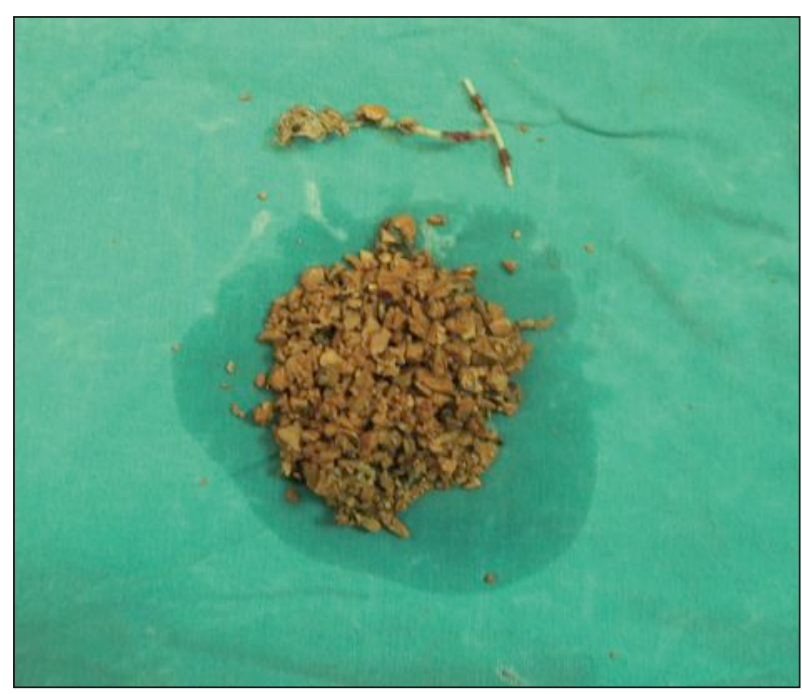

Figure 3.

Image of bladder stone at non-contrast enhanced computer tomography.

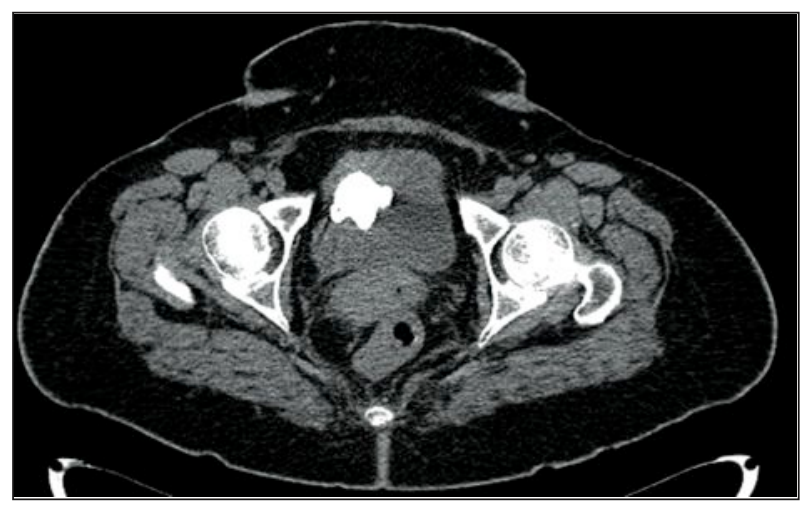

period from incontinence or contraception surgery to diagnosis of BS. All patients charts were rewieved by two author (FO and AS). Variables were defined before data collection. Microsoft Excel 2010 software (Microsoft Corporation, Redmond, WA) was used for data entry.

\section{RESULTS}

Demographic characteristics of the patients are presented in Table 1. Median age was 62 years. Six patients had undergone stress incontinance surgery including tension free vaginal tape (TVT) and trans obturator tape (TOT). One patients had an history of IUD insertion (Figure 2). All patients were symptomatic and had more than one complaint. Main complaints were dysuria (85\%), hematuria (57\%) and recurrent urinart tract infection (UTI) (57\%). Patients also reported urgency, frequency and pelvic pain. The median diagnosis time was 44.1 months (range from 9 to 218 months). Pelvic x-ray was performed all patients. To evaluate upper urinary system and perivesical area abdominal ultrasonography was also performed in 5 patients and non contrast enhanced computer tomography was performed in other 2 patients (Figure 3).

Median operative time was 41.2 minutes (range from 20 minutes to 70 minutes) and median hospitalisation time was 1.57 day (range from 1 day to 4 days). Holmium laser was used in 3 cases and pneumatic lithotripter was used in 4 cases. There was no intra operative complication. Post operative period was uneventfully for six patients. Only one patient had fever $>38^{\circ} \mathrm{C}$ on the first day of operation and ceftriaxone was started empirically at the dose of $2 \mathrm{~g}$ /day. In urine culture, ceftiraxone sensitive ESBL producing Escherichia coli was isolated. The patient was discharged at the 4th day after operation. In follow up, endoscopy was performed in six patients who underwent TUR. Cystoscopy revealed recovered bladder mucosa without protrusion of the tape into the bladder in each patient. Recurrent stress urinary incontinence was developed in two patients but both of them refused new surgical manipulation.

\section{Discussion}

Bladder stones are uncommon in women and mostly result of pathological conditions such as neurogenic bladder, bladder diverticulum and foreign material. During storage of urine a foreign body is an ideal nidus for stone formation and encrustation by calcium oxalate. Also infection in bladder accelerate the process (8). Most of foreign bodies are related with complications of urogynecologic procedures. Suture materials from bladder suspension procedures, sling procedures or IUD insertion are the most common source of an intravesical foriegn body (9).

Insertion of MUSS or IUD into the bladder leads to the development of significant symtomps and impact negatively on quality of life. Dysuria, hematuria, urgency, frequency, resistant and recurrent UTI are the most common symptoms (10). On the other hand stone formation requires time. It should be questioned why why the patients were not admitted to hospital despite they com- 
plaints and why the diagnosis was so delayed. That may be explained by socio-cultural conditions of the country. All these symptoms are mostly considered as a natural sign of aging by patients. Furthermore in rural areas its difficult to achieve health care and physicians tend to treat the symptoms without investigating underlying pathology.

To prevent further complications, it is very important to recognize intraoperatively bladder injury during stress incontinance surgery. Cystoscopy is a part of TVT procedure and the best method to evaluate the presence of bladder injury (11). Nevertheless in this paper we present five cases who underwent TVT procedure. Two hypothesis can explain the unfortunate event: sling mesh in the bladder was missed at cystoscopy or mesh was placed in the submucosal area close but outside to the bladder mucosa. Experience of surgeon can affect recognizement of bladder injury at cystoscopy. To increase the accuracy of cystoscopy, bladder must be filled with at least $300 \mathrm{~mL}$ of fluid to have better vision and use of $7^{\circ}$ or $12^{\circ}$ optics allows a more extensive view (12).

Cystoscopy is not routinely performed after TOT because of the low risk of bladder injury (13). Tayrac et al. and Abdel-Fattah et al. found less than 1\% incidence of lower urinary tract injury mostly associated with outin technique (14-15). Due to longer operative time and requirement of endoscopic instruments, cystoscopic inspection after TOT is not accepted by most surgeons. To avoid injury emptying the bladder completely is very important. We perform cystoscopy only if hematuria occurs intraoperatively or for persistance of irritative bladder symptoms.

Due to their safety and efficacy, IUDs are the most preferred method of reversible contraception all over the world (16). However insertion of IUD by paramedics and irregular follow- up evaluations can lead to serious complications such as uterine perforation (17). Harrison et al. emphased on the experience of the surgeon to prevent uterine perforation (18). After perforation, IUD could be found in any extrauterine location as rectum, omentum, peritoneum or wall of iliac vein but migration into the bladder is very rare (19-20).

Pathophysiology is still unknown but some authors believe that uterine and bladder contractions have a significant role in the migration of IUD into the bladder. Also uterin atrophy contributes to movement of IUD (21). Misplacement of IUD can cause pain, bleeding and loss of its contraception ability. In our case, the patient was 32 years-old when the T-shape IUD was placed. She had no pregnancies after insertion and symptoms were accepted as normal by the patient.

Treatment options are variable according to the experience of the physician and can be divided in open and endoscopic procedures. Open cystotomy is an alternative for big and hard stones to shorten operation time. If the mesh or IUD is very adherent and it is impossible to remove the foreign body from the bladder wall partial cystectomy may be performed (10). Pikaart et al. performed laparoscopic stone removal by following the steps of open surgery (22). With the application of technological advances in medicine, endoscopic treatments is become equally effective and more comfortable than open surgeries. Tyzortis et al. presented two case of bladder stone associated with TVT and both stones were treated endoscopically (23). Also Mustafa et al. used transurethral mesh resection and pneumotic lithotripsy for the same problem (24). Feiner et al. used Holmium laser to fragment bladder stones after TOT procedure (25). Endoscopic therapies are the first choice for us because most of bladder stones are easily fragmented. Furthermore endoscopic manipulations are not affected from body mass index of the patient and by previous surgeries.

In conclusion, misplacement of MUSS or IUD into bladder during stress incontinance surgery or contraception surgery lead to several lower urinary tract symtomps. Bladder stones are developed if the foreign material remains in the bladder. Our experience with seven cases revealed that endoscopic lithotripsy using Holmium laser and pneumotic lithotripter is a safe and effective method to manage these stones.

\section{REFERENCES}

1. Stav K, Dywer PL. Urinary bladder stones in women. Obstet Gynecol Surv. 2012; 67:715-25.

2. Papatsoris AG, Varkarakis I, Dellis A, Deliveliotis C. Bladder lithiasis: from open surgery to lithotripsy. Urol Res. 2006; 34:163-7.

3. Mwalwanda CS, Black KI. Immediate post-partum initiation of intrauterine contraception and implants: A review of the safety and guidelines for use. Aust N Z J Obstet Gynaecol. 2013; 53:331-7

4. Ulmsten U, Falconer C, Johnson P, et al.. A multicenter study of tension-free vaginal tape (TVT) for surgical treatment of stress urinary incontinence. Int Urogynecol J Pelvic Floor Dysfunct. 1998; 9:210-3.

5. Tunn R, Wildt B, Rohne J, Gauruder-Burmester A. Management of postoperative objectified intravesical position of the TVT tape two casereports. Urologe A 2006; 45:347-50

6. Irer B, Aslan G, Cimen S. Development of vesical calculi following tension-free vaginal tape procedure. Int Urogynecol J Pelvic Floor Dysfunct. 2005; 16:245-246.

7. Minaglia S, Ozel B, Klutke C. Bladder injury during transobturator sling. Urology. 2004; 64:376-377.

8. Chew R, Thomas S, Mantha ML, et al. Large urate cystolith associated with Proteus urinary tract infection. Kidney Int. 2012; 81:802-3.

9. Peyromaure M, Dayma T, Zerbib M. Development of a bladder stone following a tension-free vaginal tape intervention. J Urol. 2004; 171:337.

10. Deng DY, Rutman M, Raz S, Rodriguez LV. Presentation and management of major complications of midurethral slings: are complications under-reported? Neurourol Urodyn. 2007; 26:46-52.

11. Gold RS, Groutz A, Pauzner D, et al. Bladder perforation during tension-free vaginal tape surgery: does it matter? J Reprod Med. 2007; 52:616-8.

12. Giri SK, Drumm J, Flood HD. Endoscopic holmium laser excision of intravesical tension-free vaginal tape and polypropylene suture after anti-incontinence procedures. J Urol. 2005; 174:13061307.

13. Delorme E. Transobturator urethral suspension: mini-invasive procedure in the treatment of stress urinary incontinence in women. Prog Urol. 2001; 11:1306-1313. 
14. deTayrac R, Deffieux X, Droupy S, A prospective randomized trial comparing tension-free vaginal tape and transobturator suburethral tape for surgical treatment of stress urinary incontinence. Am J Obstet Gynecol. 2004; 190:602-628.

15. Abdel-Fattah M, Ramsay I, and Pringle S. Lower urinary tract injuries after transobturator tape insertion by different routes: a large retrospective study. BJOG. 2006; 113:1377-1381.

16. Xie ZW, Zhang YN, Wan S, et al. Levonorgestrel-releasing intrauterine device is an efficacious contraceptive for women with leiomyoma. J Int Med Res. 2012; 40:1966-72.

17. Ebel L, Foneron A, Troncoso L, et al. Intrauterine device migration to the bladder: four case reports. Actas Urol Esp. 2008; 32:530-532.

18. Harrison-Woolrych M, Ashton J, Coulter D. Uterine perforation on intrauterine device insertion: is the incidence higher than previously reported? Contraception. 2003; 67:53-56.

19. Mosley FR, Shahi N, Kurer MA. Elective surgical removal of migrated intrauterine contraceptive devices from within the peri- toneal cavity: a comparison between open and laparoscopic removal JSLS.. 2012; 16:236-41.

20. McCombie JJ, Le Fur R. Colonoscopic removal of an ectopic intrauterine device. ANZ J Surg. 2012; 82:369-70.

21. Atakan H, Kaplan M, Erturk E. Intravesical migration of intrauterine device resulting in stone formation. Urology. 2002; 60:911-913.

22. Pikaart DP, Miklos JR, Moore RD. Laparoscopic removal of pubovaginal polypropylene tension-free tape slings. JSLS. 2006; 10:220-225

23. Tzortzis V, Mitsogiannis IC, Moutzouris G. Bladderstone formation after a tension-free vaginal tape procedure: reporton two cases. Urol Int. 2007; 79:181-182.

24. Mustafa M, Wadie BS. Bladder erosion of tension-free vaginal tape presented as vesical stone; management and review of literature. Int Urol Nephrol. 2007; 39:453-5.

25. Feiner B, Auslender R, Mecz Y, et al. Removal of an Eroded Transobturator Tape from the Bladder Using Laser Cystolithotripsy and Cystoscopic Resection. Urol. 2009; 73:681.e15- 681.e16.

\section{Correspondence}

Abdulmuttalip Simsek, MD (Corresponding author)

simsek76@yahoo.com

Faruk Ozgor, MD

Mehmet Fatih Akbulut, MD

Erkan Sönmezay, MD

Omer Sanlar, MD

Ahmet Yalcin Berberoglu, MD

Zafer Gokhan Gurbuz, MD

Haseki Research and Education Hospital, Department of Urology,

Millet Cad. No: 11 - 34000 Fatih, Istanbul, Turkey

Bahar Yuksel, MD

Istanbul Medical Faculty, Gynecology and Obstetric Department

Istanbul, Turkey 